\title{
Neural network based explicit MPC for chemical reactor control
}

\author{
Karol Kiš, Martin Klaučo \\ Slovak University of Technology in Bratislava, \\ Radlinského 9, SK-812 37 Bratislava, Slovak Republic \\ karol.kis@stuba.sk
}

\begin{abstract}
In this paper, implementation of deep neural networks applied in process control is presented. In our approach, training of the neural network is based on model predictive control, which is popular for its ability to be tuned by the weighting matrices and for it respecting the system constraints. A neural network that can approximate the MPC behavior by mimicking the control input trajectory while the constraints on states and control input remain unimpaired by the weighting matrices is introduced. This approach is demonstrated in a simulation case study involving a continuous stirred tank reactor where a multi-component chemical reaction takes place.
\end{abstract}

Keywords: model predictive control, artificial neural networks, process control, continuous stirred tank reactor

\section{Introduction}

Chemical reactors play an essential role in the chemical and petrochemical industry. Their vast presence in the industrial world makes control synthesis very attractive for researchers. In recent years, several new control approaches have emerged mainly from the optimal control theory, as discussed by Smets et al. (2004); Pourdehi and Karimaghaee (2018); Bakošová et al. (2012). The optimal control theory proved to be very promising due to its natural ability to cope with technological constraints and to follow a performance criterion, which defines the overall economy of the production.

Designers of control strategies for chemical reactors must cope with several obstacles, mainly the natural instability of the process, e.g., exothermic reactors, and keeping the process variables in their designed steady state. Alongside which, the controller has to be able to decrease the energy consumption and increase the quality of the product. All control objectives can be incorporated in an optimal control problem (OCP) (Bakošová and Oravec, 2014; Singh et al., 2010; Bakošová et al., 2017). The model predictive control (MPG) technique is often used in such control tasks (Prasath et al., 2010), since its construction is straight-forward (Maciejowski, 2002).

The core concept of MPG is to predict future evolution of the controlled variables based on current measurements. Then, with respect to a quality criterion (usually energy consumption), MPC optimizes the values of the manipulated variables so that the criterion is minimized (Klaučo and Kvasnica, 2019). Even though it seems that MPG is one of the best controllers, it has several drawbacks. Since it is an optimization-based controller, it requires a repeated solution to an optimal control problem. Such an arrangement is virtually impossible to implement in the industry or on the computers responsible for the chemical reactor operation.

Traditional way of coping with this limitation is to consider explicit model predictive control (EMPC), an analytical solution to the optimal control problem (Bemporad et al., 2002). The control law given by the explicit solution is in form of the piecewise affine function (PWA) (Borrelli et al., 2017). Such control law can be easily evaluated at any given time without the need to involve an optimization procedure. In other words, it allows replacing the optimization solver with function evaluation. EMPC, unfortunately, can be constructed only for small-sized systems with short prediction horizons, which is a significant limitation in process industries, where prediction horizons are long.

This paper proposes an alternative to the explicit controller based on neural networks. The neural network is a powerful mathematical concept capable of approximating an arbitrary continuous function (Hornik, 1991). Here, approximation of the explicit control law given by the fullfidelity MPC is proposed. Since the traditional explicit model predictive control resulting in PWA function is not considered, the length of the prediction horizon nor the size of the controlled system are not limiting factors. Similar work has been done by Karg and Lucia (2018) or by Lohr et al. (2019). In this paper, however, we focus on the application in chemical technology, mainly the control of multicomponent chemical reaction.

\section{Theoretical}

Firstly, optimal control problem (OCP) that stands for the model predictive controller is presented. 
The second part of this section is devoted to the artificial neural network to substitute the model predictive controller.

\section{Model Predictive Control}

Standard formulation of the model predictive controller utilizes a linear time-invariant model that captures the dynamics of the controlled process. Specifically, discrete-time dynamics were considered:

$$
\begin{gathered}
x\left(t+T_{S}\right)=A x(t)+B u(t), \\
y(t)=C x(t)+D u(t),
\end{gathered}
$$

where $x \in \mathbb{R}^{n \mathrm{x}}$ stands for process state variables, vector $u \in \mathbb{R}^{n u}$ represents manipulated variables and $y \in \mathbb{R}^{n u}$ depicts the output variable. Matrices $A \in \mathbb{R}^{n \times \times n \mathrm{x}}, B \in \mathbb{R}^{n \times \times n u}, C \in \mathbb{R}^{n y \times n \times}$, and $D \in \mathbb{R}^{n y \times n u}$ were obtained from a dynamical model representing an actual controlled process using the first order Taylor expansion. The discrete time linear model (1) was discretized with the sampling period of $T_{S}$. The model predictive control was then constructed as follows

$$
\begin{gathered}
\min _{u_{0}, \ldots, u_{N-1}} \sum_{k=0}^{N-1} y_{k}^{\mathrm{T}} Q y_{k}+\sum_{k=0}^{N-1} u_{k}^{\mathrm{T}} R u_{k}, \\
\text { s. t. } x_{k+1}=A x_{k}+B u_{k}, k=1, \ldots, \mathrm{N}-1, \\
y_{k}=C x_{k}+D u_{k}, k=1, \ldots, \mathrm{N}-1, \\
x_{0}=x(t) \\
y_{\min } \leq y_{k} \leq y_{\max }, k=1, \ldots, \mathrm{N}-1, \\
u_{\min } \leq u_{k} \leq u_{\max }, k=1, \ldots, \mathrm{N}-1,
\end{gathered}
$$

where $N$ denotes the prediction horizon. Cost function (2a) is in form of convex quadratic function with positive definite tuning factors $Q \in \mathrm{R}^{n \times x n x}$ and $R \in \mathrm{R}^{n u \times m u}$. The objective function is posed so that the controlled variables are driven towards the steady-state. Moreover, technological constraints were defined as min-max limits on controlled as well as on manipulated variables, as in (2e) and (2f), respectively. The optimization problem is initialized with the measurement of $x(t)$. MPC is formulated as a quadratic optimization problem with linear constraints. Optimal solution to the MPC in (2) yields an optimal sequence of manipulated variables $\left[u_{0}^{*}\right.$, $\left.\ldots, u_{\mathrm{N}-1}^{*}\right]^{\mathrm{T}}$. Since OCP is a convex optimization problem, its solution is a global minimum.

The process is controlled by the model predictive controller using an algorithm called receding horizon policy, presented and proven by Mayne et al (2000):
1. Measure system process variables $x(t)$ (e.g. temperature or concentration).

2. Initialize MPC in (2) with $x(t)$.

3. Solve quadratic optimization problem.

4. Apply manipulated variable $u_{0}$.

5. After $T_{S}$ continue from step 1 .

We refer to this algorithm as to a closed-loop implementation of MPC. The bottleneck of the algorithm is step No. 3, where an optimization problem has to be solved. In average industrial applications, this is an impossible task since there are no machines capable of solving a complex mathematical problem, and the solver imposes additional costs.

In the next section, replacement of such an algorithm with neural network is discussed, in order to substitute the complex procedure of mathematical optimization performed with every sampling.

\section{Artificial Neural Networks}

A neural network is a mathematical function that maps inputs $z \in \mathbb{R}^{n z} \rightarrow w \in \mathbb{R}^{n w}$ via interconnected monotone functions. Structure of the neural network is visualized in Figure 1, where each green and red dot represents the monotone function, also called as activation functions, and they are given as

$$
\varphi(\alpha, z)=\frac{2}{1+\mathrm{e}^{\alpha z}}-1,
$$

where $z$ denotes an aggregated input to each node while $\alpha$ is a tuning parameter of the activation function. Blue dots stand for linear output layer.

Neural network is capable of approximating any arbitrary continuous functions with a very high confidence number, as discussed by Hornik (1991). Another significant advantage is the explicit nature of the neural net. Once a neural net of suitable properties is constructed, it can be evaluated on moderate hardware and hence no optimization solver is needed. Another main advantage is that the neural net is in no way limited by the length of the prediction horizon or by the size of the system, as it is in explicit model predictive control. The structure of an NN-based controller with $\alpha$ values determines the goodness of the PWA control law approximation. The procedure of getting weights is called training of the neural network and it is performed for a fixed structure of the neural net and thus, only the weights are calculated.

To give the reader an illustration on how the weights are calculated, consider

$$
\min _{\alpha} \sum_{k=1}^{n_{k}}\left(\tilde{w}_{k}-\varphi\left(\alpha, z_{k}\right)\right)^{2} .
$$

The optimization problem (4) is a sum-of-squares data fitting problem. Solution to (4) gives opti- 


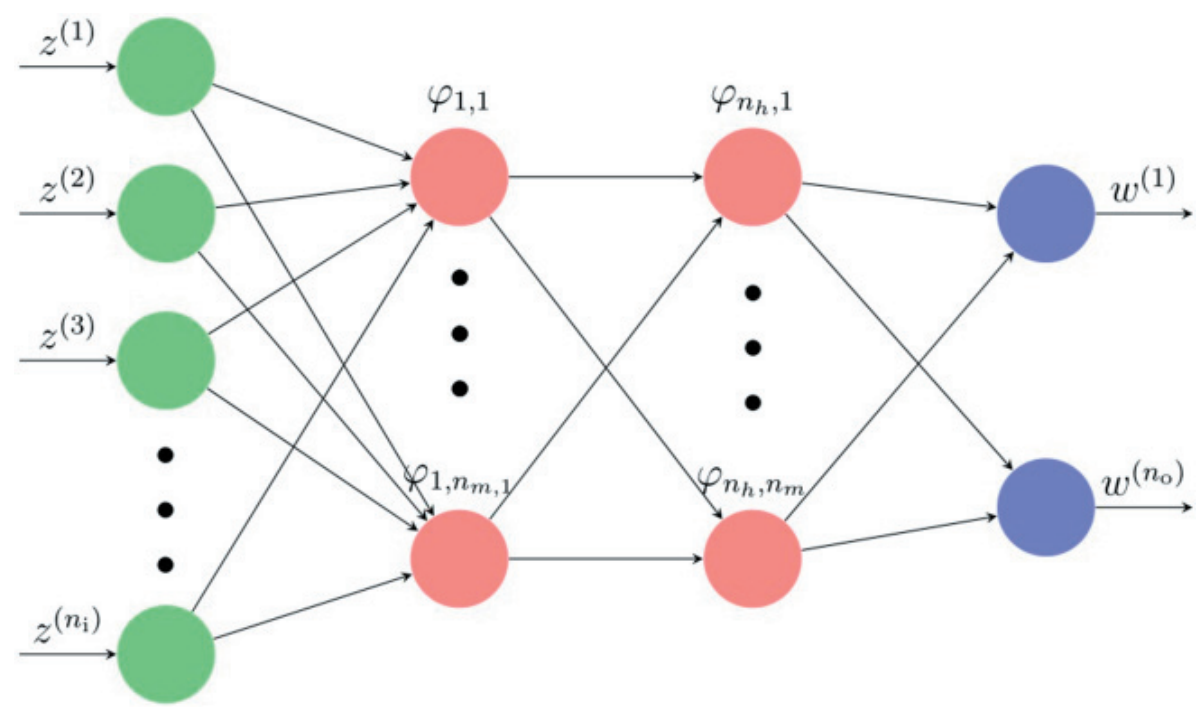

Fig. 1. Example of the neural network structure. Green points represent the input layer, red dots depict hidden layers, and the output layer is blue.

mal values of weight $\alpha$ based on minimizing the squared distance between a target value, $\tilde{w}$, and the evaluated activation function, $\varphi\left(\alpha, z_{k}\right)$, for a given neural network input. Naturally, to increase the goodness of the $\alpha$ value, a sufficient number of data points has to be included in the minimization procedure. Here, $n_{k}$ denotes the number of these data points.

Since the neural network substitutes the controller, measurements of the process variables $x(t)$ are the input to the training of the NN-based controller, and control inputs, i.e., the manipulated variable $u(t)$, are the training targets. To provide a suitable basis for the training procedure, an initial training set was given as

$$
\mathcal{X}=\left[x_{\min }, \ldots, x_{\max }\right],
$$

where the limits on the process variables with $n_{k}$ points form an equidistant grid. Subsequently, for each data point in $\mathcal{X}$, a corresponding control action was calculated and a set $\mathcal{U}$ with $n_{k}$ different manipulated variables was obtained. The corresponding control actions were obtained by solving the MPC problem (2) initialized with $x_{0}$ from (2g) equal to one of the points from $\mathcal{X}$. Sets $\mathcal{X}$ and $\mathcal{U}$ together form a learning data set, from which the minimization problem (4) is constructed. Note that problem (4) is presented only for one node in the hidden layer, the overall training procedure consists of an aggregated minimization problem, where all nodes are included.

Numerically, the optimal control actions are obtained using the GUROBI solver, while the training procedure is performed with the Deep Learning Toolbox in MATLAB. Furthermore, the model pre- dictive controller is formulated using the YALMIP toolbox (Lofberg, 2004).

\section{Experimental}

The theory presented in the Theoretical part of the paper was applied in a case study involving the control of a multi-component chemical reactor. Specifically, we considered benchmark chemical reaction

$$
\mathrm{A} \rightleftarrows \mathrm{B} \rightarrow \mathrm{C},
$$

with dynamical behavior given by three differential equations (Fissore, 2008; Bakaráč and Kvasnica, 2018) leading to

$$
\begin{gathered}
\dot{c}_{A}=-k_{1} c_{\mathrm{A}}+\frac{F}{V}\left(c_{\mathrm{A}, \text { feed }}-c_{\mathrm{A}}\right)-k_{2} c_{\mathrm{C}}^{2}, \\
\dot{c}_{B}=-\frac{F}{V} c_{\mathrm{B}}-k_{3} c_{\mathrm{C}}^{2}, \\
\dot{c}_{C}=k_{1} c_{\mathrm{A}}+\frac{F}{V} c_{\mathrm{C}}-\left(k_{2}+k_{3}\right) c_{\mathrm{C}}^{2}+q_{i n} .
\end{gathered}
$$

Process variables are concentrations $x=\left[c_{\mathrm{A}}, c_{\mathrm{B}}, c_{\mathrm{C}}\right]$ ', while the manipulated variables, $q_{i n}$, stands for molar feed of component $C$. Specific parameters of the benchmark model of chemical reactor are reported in Table 1. Objective of the controller was to keep the concentration, $c_{B}$, at a steady state level, which represents the optimal conditions of the reactor operation as introduced by Fissore (2008).

Concretely, the optimal operation of the chemical reactor is given by a set of steady-state values of individual process variables, and they are given as 
Tab. 1. Table of model parameters.

\begin{tabular}{lcc}
\hline Variable & Value & Unit \\
\hline$k_{1}$ & 1 & $\mathrm{~m}^{3} \mathrm{~mol}^{-1} \mathrm{~s}^{-1}$ \\
$k_{2}$ & 3 & $\mathrm{~m}^{3} \mathrm{~mol}^{-1} \mathrm{~s}^{-1}$ \\
$k_{3}$ & 5 & $\mathrm{~m}^{3} \mathrm{~mol}^{-1} \mathrm{~s}^{-1}$ \\
$F$ & 3 & $\mathrm{~m}^{3} \mathrm{~s}^{-1}$ \\
$V$ & 3 & $\mathrm{~m}^{3}$ \\
$c_{\mathrm{A}, \text { feed }}$ & 2 & $\mathrm{~mol} \mathrm{~m}^{-3}$ \\
\hline
\end{tabular}

$$
\begin{gathered}
c_{A, S}=2.18 \mathrm{~mol} \mathrm{~m}^{-3}, c_{B, S}=3.93 \mathrm{~mol} \mathrm{~m}^{-3}, \\
c_{C, S}=0.87 \mathrm{~mol} \mathrm{~m}^{-3},
\end{gathered}
$$

while the steady-state manipulated variables were set to $q_{i n, S}=5 \mathrm{~mol} \mathrm{~s}^{-1}$.

Next, the model predictive controller was constructed with linearized version of the dynamical mathematical model, sampled with $T_{S}=0.1 \mathrm{~s}$.

Matrices of the discretized state-space model take the form:

$$
\begin{aligned}
A & =\left[\begin{array}{ccc}
0.83 & 0 & 0.24 \\
0.03 & 0.90 & 0.43 \\
0.05 & 0 & 0.23
\end{array}\right], \\
\mathbf{B} & =\left[\begin{array}{l}
0.02 \\
0.03 \\
0.05
\end{array}\right], \quad \mathrm{C}=\left[\begin{array}{lll}
0 & 1 & 0
\end{array}\right] .
\end{aligned}
$$

MPC was set up with the prediction horizon $N=50$, while the tuning factors were set to $Q=10$ and $R=0.15$. The constraints were again reproduced from the benchmark model where $0 \leq c_{\mathrm{A}} \leq 10$, $0 \leq c_{\mathrm{B}} \leq 14$, and $0 \leq c_{\mathrm{C}} \leq 1.1$. Molar feed-flow of $q_{\text {in }}$ was constrained to the interval of $[0,10] \mathrm{mol} \mathrm{s}^{-1}$. Learning set for the neural network was then constructed. Each interval for the process variables was split into 10000 samples, for which the corresponding optimal value of the manipulated variable was determined. Such a training set was then fed into the Deep Learning Toolbox, particularly, the fitnet com-
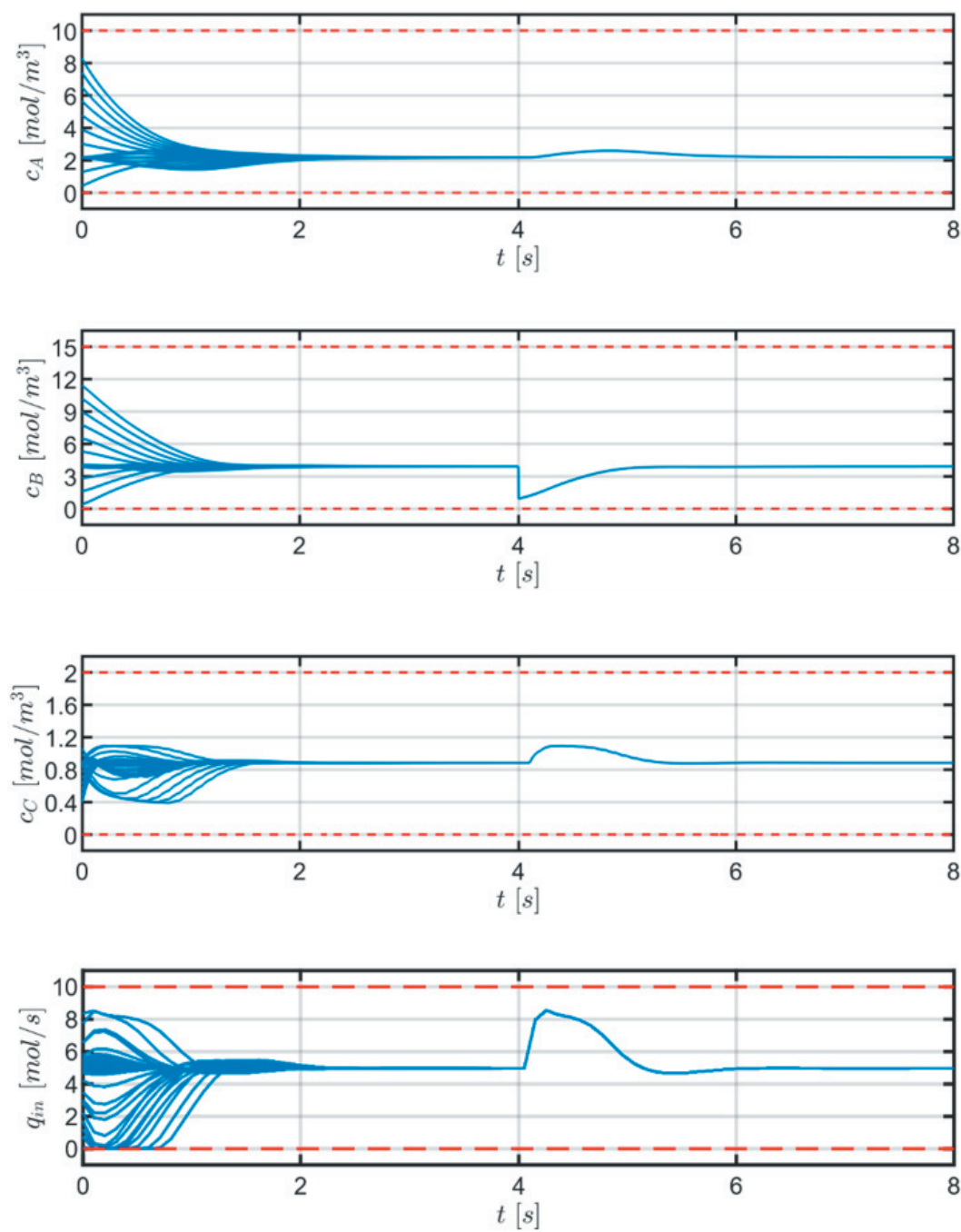

Fig. 2. Control performance of the NN-based controller with various initial conditions. 
mand, which trained the neural network. Structure of the NN-based controller consists of three nodes in the input layer (due to three individual measurements of the process variable) and from four hidden layers. Each hidden layer consists of four nodes, each in form of the action function, as in (3). The final output layer has linear structure and consists of one node. Recall that the output from the neural network is the manipulated variable. The training was done offline, and it took $80 \mathrm{~s}$ on a personal computer with Core i7, 16 GB of RAM, and Matlab R2019a. The resulting neural network controller takes less than $7 \mathrm{kB}$ of memory and can be evaluated in milli-second range on ARM processors. Such a characteristic is in strong contrast to the optimization procedure required by the MPC strategy. Finally, applicability of the NN-based controller was evaluated and tested. A large scale test scenario was prepared, involving 600 simulations, each starting from a different initial condition. Thus, the performance of the NN-based controller can be easily observed. Furthermore, artificial disturbance to the controller variable was introduced to present that the NN-based controller can also effectively cope with disturbances.

Adherence of the bounds on the process and manipulated variables has to be especially pinpointed. Control scenarios can be seen in Figure 2, but only a subset of the 600 simulations is shown to make the figure readable. Also, all simulations are performed using the full-fidelity non-linear model presented in $(7)$.

Even though the performance of the NN-controller is, in terms of the

$$
J=\sum_{t=0}^{t_{\text {sim }}}\left\|x(t)-x_{S}\right\|_{2}^{2},
$$

simulations, satisfactory, a quality criterion of the following form was also evaluated to indicate how far the actual measurement of process variables was from the desired steady-state value. For all 600 simulations, the $J$ value was evaluated and compared with the value of the criterion from the model predictive control performance. The worst decrease in the suboptimality was $2.14 \%$.

\section{Conclusions}

The paper discussed the design of suboptimal control law in form of a neural network. The main advantage of this controller is its explicit form, which was constructed for a large prediction horizon. The neural network was constructed based on data obtained from the optimal solution to a full-fidelity model predictive controller. Applicability of the suboptimal controller was tested on a large-scale simulation case study involving the stabilization of multi-component chemical reaction. Simulation results showed that in $94.5 \%$ of cases, the NN-based explicit controller performed with the optimality decrease below $1 \%$.

\section{Acknowledgments}

The authors gratefully acknowledge the contribution of the Scientific Grant Agency of the Slovak Republic under the grant 1/0585/19. This work was supported by the funding of the Slovak Ministry of Education, Science, Research and Sport under the project STU as the Leader of Digital Coalition 002STU-2-1/2018. M. Klaučo would like to thank for the financial contribution from the STU Grant Scheme for Excellent Research Teams.

\section{References}

Bakaráč P, Kvasnica M (2018) Fast nonlinear model predictive control of a chemical reactor: a random shooting approach. Acta Chimica Slovaca, 11(2): 175-181.

Bakošová M, Mészáros A, Klemeš J, Oravec J (2012) Robust and optimal control approach for exothermic reactor stabilization. Theoretical Foundations of Chemical Engineering, (46): 740-746.

Bakošová M, Oravec J, Mészáros A, Vasičkaninová A (2017) Neural-Network-Based and Robust ModelBased Predictive Control of a Tubular Heat Exchanger. Chemical Engineering Transactions, (61): 301-306.

Bakošová M, Oravec J (2014) Robust mpc of an unstable chemical reactor using the nominal system optimization. Acta Chimica Slovaca, 7(2): 87-93.

Bemporad A, Morari M, Dua V, Pistikopoulos EN (2002) The explicit linear quadratic regulator for constrained systems. Automatica, 38(1): 3-20.

Borrelli F, Bemporad A, Morari M (2017) Predictive Control for Linear and Hybrid Systems. Cambridge University Press.

Fissore D (2008) Robust control in presence of parametric uncertainties: observer-based feedback controller design. Chemical Engineering Science, 63(7): 18901900.

Hornik K (1991) Approximation capabilities of multilayer feedforward networks. Neural Networks, 4(2): 251-257.

Karg B, Lucia S (2018) Efficient representation and approximation of model predictive control laws via deep learning.

Klaučo M, Kvasnica M (2019) MPC-Based Reference Governors. Springer, $1^{\text {st }}$ edition.

Lofberg J (2004) YALMIP: A Toolbox for Modeling and Optimization in MATLAB. In Proc. of the CACSD Conference, Taipei, Taiwan. Available from http:// users.isy.liu.se/johanl/yalmip/.

Lohr Y, Klaučo M, Kalúz M, Monnigmann M (2019) Mimicking predictive control with neural networks in domestic heating systems. In Fikar $\mathrm{M}$ and Kvasnica $\mathrm{M}$, editors, Proceedings of the $22^{\text {nd }}$ International Conference on Process Control, pages 19-24, Šrbské Pleso, Slovakia. Slovak University of Technology in Bratislava, Slovak Chemical Library. 
Maciejowski JM (2002) Predictive Control with Constraints. PEARSON Prentice-Hall.

Mayne DQ, Rawlings JB, Rao CV, Scokaert POM (2000) Constrained model predictive control: Stability and optimality. Automatica, 36(6): 789-814.

Pourdehi S, Karimaghaee P (2018) Stability analysis and design of model predictive reset control for nonlinear time-delay systems with application to a two-stage chemical reactor system. Journal of Process Control, 71: 103-115.

Prasath G, Recke B, Chidambaram M, Jørgensen J (2010) Application of Soft Constrained MPC to a Cement Mill Circuit. In Proceedings of the $9^{\text {th }}$ International Symposium on Dynamics and Control of Process Systems, Belgium, Leuven.
Singh A, de Villiers P, Rambalee P, Gous G, de Klerk J, Humphries G (2010) A holistic approach to the application of model predictive control to batch reactors. IFAC Proceedings Volumes, 43(9): 127-132. $13^{\text {th }}$ IFAC Symposium on Automation in Mining, Mineral and Metal Processing.

Smets IY, Claes JE, November EJ, Bastin GP, Impe JFV (2004) Optimal adaptive control of (bio)chemical reactors: past, present and future. Journal of Process Control, 14(7): 795-805. Dynamics, Monitoring, Control and Optimization of Biological Systems. 